\title{
Impact of everolimus on survival after liver transplantation for hepatocellular carcinoma
}

\author{
Incheon Kang ${ }^{1}$, Dong Jin $\mathrm{Joo}^{2}$ \\ 'Division of Transplantation, Department of Surgery, CHA Bundang Medical Center, Seongnam, Korea \\ ${ }^{2}$ Division of Transplantation, Department of Surgery, Yonsei University College of Medicine, Seoul, Korea
}

Background: The objective of this study was to investigate whether everolimus (EVR) use affects long-term survival after liver transplantation (LT) in patients with hepatocellular carcinoma (HCC).

Methods: Data from 303 consecutive patients with HCC who underwent LT were retrospectively reviewed. The patients were divided into two groups: (1) patients treated with EVR in combination with calcineurin inhibitors (CNI) (EVR group, $n=114$ ), and (2) patients treated with CNI-based therapy without EVR (non-EVR group, $n=189$ ). Disease-free survival (DFS) and overall survival (OS) were compared between two groups, and prognostic factors for DFS and OS were evaluated.

Results: The EVR group exhibited more aggressive tumor biology than the non-EVR group, including a higher number of tumors $(P=0.003)$, a higher prevalence of microscopic vascular invasion $(P=0.017)$, and cases exceeding Milan criteria $(P=0.029)$. Compared with the non-EVR group, the EVR group had significantly better DFS $(P=0.029)$ and OS $(P<0.001)$. In multivariate analysis, use of EVR was identified as an independent prognostic factor for DFS (hazard ratio [HR], 0.248; P=0.001) and OS (HR, 0.145; $\mathrm{P}<0.001)$.

Conclusions: In combination with CNI, EVR has the potential to prolong long-term survival in patients undergoing LT for HCC. These findings warrant further investigation in a well-designed prospective study.

Corresponding author: Dong Jin Joo

E-mail: djjoo@yuhs.ac

(C) The Korean Society for Transplantation

This is an Open Access article distributed under the terms of the Creative Commons Attribution Non-Commercial License (http://creativecommons.org/licenses/by-nc/4.0/) which permits unrestricted non-commercial use, distribution, and reproduction in any medium, provided the original work is properly cited. 